\title{
Positive Impact of Intraoperative Epidural Ropivacaine Infusion on Oncologic Outcomes in Pancreatic Cancer Patients Undergoing Pancreatectomy: A Retrospective Cohort Study
}

\author{
Wannan Chen ${ }^{1^{*}}$, Yaolin $\mathrm{Xu}^{2^{*}}$, Yueming Zhang2 ${ }^{2}$, Wenhui Lou ${ }^{\circledR}$ and Xiaodan $\operatorname{Han}^{1 凶}$ \\ 1. Department of Anesthesiology, Zhongshan Hospital Fudan University, 180 Fenglin Road, Shanghai, China. \\ 2. Department of General Surgery, Zhongshan Hospital Fudan University, 180 Fenglin Road, Shanghai, China \\ *These authors contributed equally to this research and should be considered as co-first authors. \\ Corresponding authors: Wenhui Lou, E-mail: lou.wenhui@zs-hospital.sh.cn, +86-18616881868; Xiaodan Han, E-mail: han.xiaodan@zs-hospital.sh.cn, \\ +86-13761223509, Fax number: + 86-21-64043947. \\ (c) The author(s). This is an open access article distributed under the terms of the Creative Commons Attribution License (https://creativecommons.org/licenses/by/4.0/). \\ See http://ivyspring.com/terms for full terms and conditions.
}

Received: 2020.12.29; Accepted: 2021.05.09; Published: 2021.05.27

\begin{abstract}
Background: Previous literatures have demonstrated that regional anesthesia such as epidural anesthesia may affect long-term survival of cancer patients. In the present study, we conducted a retrospective cohort study to investigate the survival impact of intraoperatively epidural ropivacaine infusion on pancreatic ductal adenocarcinoma (PDAC) patients.

Methods: PDAC patients who underwent pancreatic surgery in Zhongshan Hospital Fudan University from January, 2015 to June, 2018 were included. The surgical procedure was performed under combined endotracheal general anesthesia and thoracic epidural anesthesia, and patient-controlled epidural analgesia (PCEA) with $0.12 \%$ ropivacaine was given after surgery for further pain control. Patients were divided into two groups according to their intraoperative epidural ropivacaine concentration: high $(0.375 \%-0.5 \%)$ and low (0.15\%-0.25\%). Survival outcome was compared between groups.

Results: A total of 215 patients were enrolled and their baseline characteristics were balanced between groups, except that patients with high concentration ropivacaine received higher total dose opioid and had longer operative time. Resected PDAC patients who were administrated with high concentration ropivacaine through epidural catheter intraoperatively had improved overall survival (median overall survival, mOS, high VS low, 37.6 VS 23.7 months, $p=0.04$ ). High epidural ropivacaine concentration was an independent prognostic factor (hazard ratio $[H R]=0.65,95 \%$ confidence interval $[\mathrm{Cl}], 0.44-0.94 ; p=0.03$ ). Subgroups analyses shown that T3M0 PDAC patients with preoperative CA 19-9 higher than $200 \mathrm{U} / \mathrm{ml}$, negative resection margin, and those without tumor deposit and adjuvant radiotherapy could benefit from high concentration of ropivacaine.

Conclusion: Intraoperatively epidural infusion with high concentration of ropivacaine was associated with improved OS in PDAC patients undergoing pancreatectomy.
\end{abstract}

Key words: pancreatic ductal adenocarcinoma, epidural anesthesia, ropivacaine, overall survival, pancreatectomy

\section{Introduction}

Pancreatic ductal adenocarcinoma (PDAC) is high malignant tumor with dismal prognosis, causing approximately 85,000 and 40,000 death per year in China and the United States [1]. Without obvious early symptoms, majority of patients are diagnosed in advanced stage and lost chance of curative surgery.
As a result, the five-year survival rate remains at only $9 \%[2]$.

Currently, surgical resection is only possible curative treatment for pancreatic cancer. However, surgical intervention might impair cell-mediated immunity and activated circulating tumor cells 
implantation which leave patients susceptible to develop of postoperative recurrence or metastasis $[3,4]$. Multiple retrospective studies have shown that regional anesthesia improved patient outcome after surgery in bladder, breast and lung cancers [5-7]. In addition, regional anesthesia such as epidural anesthesia may blunt the pro-metastatic effect of surgical stress and inhibit tumor growth and metastasis, especially in high concentrations [8]. Tyler et al. also observed that perioperative epidural analgesia was a positive prognostic factor in patients undergoing resection of pancreatic cancer [9].

Ropivacaine was a frequently-used long-acting amide regional anaesthetic agent. Baptista-Hon demonstrated that ropivacaine decreases the ability of cell invasion in colon cancer by regulating NaV1.5 channel activity [10]. Meanwhile, ropivacaine could inhibit lung and breast cancer cell proliferation by arresting cell cycle in vitro [6,7]. However, it is unclear whether regional anesthesia with ropivacaine during pancreatic surgery was associated with any improvement in oncologic outcomes. Thus, we conducted this retrospective study to investigate whether there is association between intraoperative epidural ropivacaine concentration and long-term clinical outcome of PDAC patients.

\section{Materials and Methods}

\section{Study Population}

We enrolled PDAC patients who received curative resection of primary pancreatic cancer in our institution from January, 2015 to June, 2018. All the enrolled patients were selected based on the following criteria: 1) aged 18 years or older, 2) with pathologically confirmed evidence of PDAC, 3) without history of other primary malignant tumor, 4) no history of antitumor treatment before surgery, 5) received combined general-epidural anesthesia, 6)

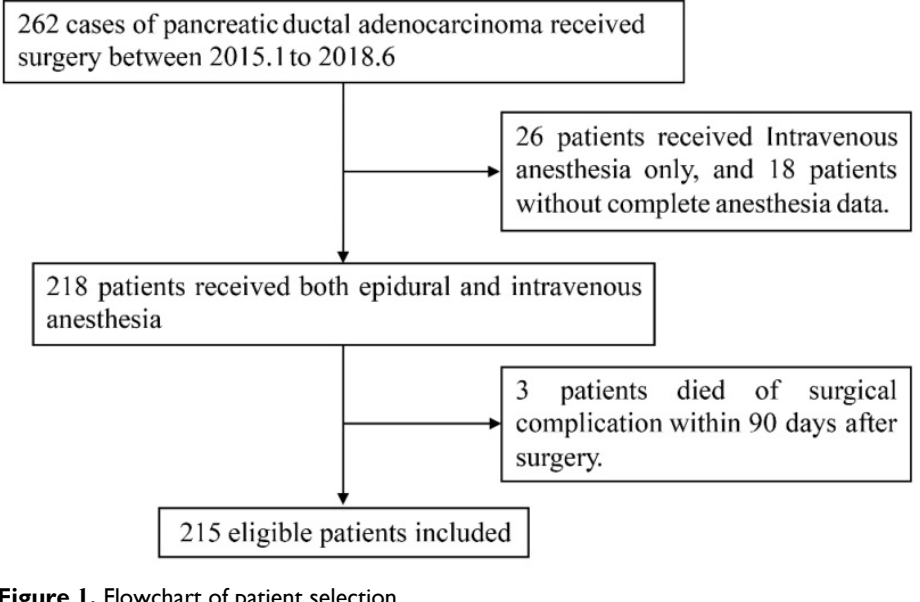

Figure 1. Flowchart of patient selection. with complete clinicopathologic data. Patients who died within 90 days after surgery because of postoperative complications were not able to evaluate their long-term oncologic outcome and thus were excluded from the study. A flowchart of patient selection was shown in Figure 1.

\section{Study Design}

In the present study, we aimed to evaluate whether intraoperative epidural ropivacaine infusion was associated with long-term oncology outcome of PDAC patients. The primary endpoint was overall survival (OS). OS was defined as the period from the date of surgery to the date of death or the last follow-up. The secondary endpoint was disease free survival (DFS), which was defined as time from surgery to either local recurrence or distant metastasis occurred. The latest follow-up was performed in October, 2020. The survival data was obtained from medical records and follow-up telephone interviews.

This study was approved by the Ethics Board of Zhongshan Hospital, Fudan University (B2020-409R). Informed consent was waived because the data was anonymized before analysis.

\section{Clinicopathologic Data}

Electronic medical records of recruited patients, including demographic characteristics, pathologic results, preoperative serum parameters, and the American Society of Anesthesiologists (ASA) physical status classification, were collected from the database of Zhongshan Hospital, Fudan University clinical information system. The data of American Joint Committee on Cancer (AJCC) $8^{\text {th }}$ TNM stage, tumor differentiation, microvascular invasion (MVI), peripancreatic fat invasion (FI), neural invasion (NI), surgical margin, and tumor deposit were extracted from final pathologic reports. A positive resection margin was defined as either microscopic or macroscopic presence of tumor cells at definite resection margins. Tumor deposit was a microscopic or macroscopic tumor nodule located in the peripancreatic fat or lymphatic drainage bed of pancreatic cancer. A carbohydrate antigen 19-9 (CA 19-9) cut point of $35 \mathrm{U} / \mathrm{ml}$ was set to dichotomize patients with normal and elevated values based on a research of Aldakkak and colleagues. Patients with an elevated CA 19-9 level were then further stratified into low (36-200) and high (>200) groups [11].

\section{Exposure Variable}

The aim of the study was to observe the effect of intraoperative epidural ropivacaine concentration on clinical outcomes after 
pancreatic surgery. Patients in low concentration group received $0.15 \%-0.25 \%$ ropivacaine through the epidural catheter intraoperatively, while those who received $0.375 \%-0.5 \%$ ropivacaine were belonged to high concentration group.

The anesthesia manipulation was conducted according to the attending anesthesiologist's discretion. Since high concentration of epidurally administrated ropivacaine may cause a significant hemodynamic change[12,13], anesthesiologists in our medical center prefer to delivered lower concentration of ropivacaine in elder patients for more stable hemodynamic and to reduce potential perioperative cardiovascular complications Meanwhile, higher epidural ropivacaine concentration provided not only regional analgesia but also a better relaxation of abdominal muscles [14-16]. Thus, anesthesiologists in our medical center prefer to administrate high dose of ropivacaine in patients who received radical, complicated and time-consuming surgeries, such as pancreaticoduodenectomy in the present study.

\section{Anesthesia Care}

All the intraoperative care was provided by the consultant anesthesiologists. Upon entering the operating room, patients were received epidural puncture at the mid-thoracic level (T8-T10). The procedures were done under endotracheal general anesthesia with epidural anesthesia. General anesthesia was induced with sufentanil (0.3-0.5 $\mu \mathrm{g} / \mathrm{kg}$ ), propofol (target-controlled infusion, effect-site concentration: $3.0-4.0 \mu \mathrm{g} / \mathrm{kg}$ ), and rocuronium $(0.6 \mathrm{mg} / \mathrm{kg})$. Anesthesia was maintained with desflurane (0.8MAC) in an oxygen/air mixture. Ventilator was set as follows: tidal volume $8 \mathrm{ml} / \mathrm{kg}$, respiratory rate $10-12 / \mathrm{min}$, keeping $\mathrm{EtCO} 2$ between $35-45 \mathrm{mmHg}$. Either sufentanil or fentanyl and rocuronium were given repeatedly as necessary throughout the surgery. For epidural anesthesia, all patients received a stress volume $(8-10 \mathrm{ml})$ of $0.1875-0.5 \%$ ropivacaine before the incision and a bolus volume (4-5 ml) each hour. Patients were sent to post anesthesia care unit (PACU) for a further one-hour monitored care. Per the empirical therapy in our center, all patients received a patient-controlled epidural analgesia (PCEA) pump $(0.12 \%$ ropivacaine and $0.4 \mu \mathrm{g} / \mathrm{ml}$ sufentanil, background: $3 \mathrm{ml} /$ hour, bolus $4 \mathrm{ml} /$ hour, lockout time: $10 \mathrm{~min}$ ) to control the postoperative pain. The dose conversion ratio for fentanyl/sufentanil was 6 based on previous research and the total opioid dose was defined as intraoperative opioid dose and calculated as relative total fentanyl administration [17].

\section{Statistical Analysis}

Data analyses were conducted and graphics were generated by using $\mathrm{R}$ project 4.04 (heep://www.r-project.org/) for Windows and IBM SPSS Statistics 22.0 version. Categorical variables were reported as frequencies and percentages, while continuous variables were described as medians. Normality and homogeneity of variance were tested using Shapiro-Wilk test and Levene's test. T-tests or Mann-Whitney $U$ tests were used to compare continuous variables and Fisher's exact test or Pearson's chi-squared test was used to analyze categorical variables between groups. The Cox proportional hazards model was used to estimate the hazard ratio (HR) of death. The significant statistical variables $(p<0.05)$ in univariate Cox regression analysis were included into multivariate analysis using a forward conditional method to identify the independent prognostic factors for survival. Kaplan-Meier (KM) method was used to calculate OS, DFS, and follow-up period. Exploratory subgroup analyses were conducted to investigate the potential effect of epidural ropivacaine infusion on oncologic outcomes in certain subgroups and were illustrated by forest plot.

\section{Results}

\section{Study population characteristics}

A total of 215 PDAC patients were included in the present study and the clinicopathologic characteristics were summarized in Table 1. The proportion of patients with higher and lower concentration of ropivacaine infusion was 55.3\% $(n=119)$ and $44.7 \%(n=96)$, respectively. None of the baseline characteristics differed significantly between two groups except for total opioid dose (high VS low, 198.9 VS $180 \mu \mathrm{g}$ fentanyl, $\mathrm{p}=0.049$ ) and operation duration (high VS low, 239.3 VS $187.1 \mathrm{~min}, \mathrm{p}<0.001$ ). Meanwhile, we found that younger male patients who accepted pancreatoduodenectomy were more likely to receive higher dose of epidural ropivacaine infusion intraoperatively (high VS low; male, $63.9 \%$ VS 51\%, $\mathrm{p}=0.071$; mean age, 62.7 VS $64, \mathrm{p}=0.21$; pancreatic head lesion, $59.7 \%$ VS $47.9 \%, p=0.083$ ), although the difference were insignificant.

\section{Epidural anesthesia with higher concentration of ropivacaine was associated with better OS in PDAC patients}

In this study, median follow-up time for whole cohort was 38.3 months (95\% confidence interval, 95\% CI, 37.2 to 39.7). Survival analysis (Fig. 2) demonstrated that high dose intraoperative epidural ropivacaine infusion was associated with improved 
OS in PDAC patients (median OS, mOS, high VS low, 37.6 VS 23.7 months, $p=0.04$ ). Although it was statistically insignificant, we also observed a better DFS in high concentration group (median DFS, mDFS, high VS low, 24.1 VS 15.6 months, $\mathrm{p}=0.052$ ). Cox proportional hazards models was constructed to assess association of relevant variables with OS and was summarized in Table 2. Univariate Cox regression analysis indicated that $\mathrm{T}$ stage $(\mathrm{p}<0.01), \mathrm{N}$ stage $(p<0.01), \quad M$ stage $(p=0.019)$, adjuvant chemotherapy $(p=0.04)$, preoperative CA 19-9 level $(p<0.01)$, NI $(p<0.01)$, FI $(p<0.01)$, tumor deposits $(p<0.01)$, tumor differentiation $(p<0.01)$, and epidural ropivacaine concentration $(p=0.041)$ were all significantly associated with OS. Further multivariate Cox regression analysis confirmed $\mathrm{T}$ stage $(\mathrm{p}<0.01), \mathrm{N}$ stage $(p<0.01), \quad M$ stage $(p<0.01)$, adjuvant chemotherapy $(p=0.04)$, preoperative CA 19-9 level $(\mathrm{p}<0.01)$, tumor differentiation $(\mathrm{p}=0.042)$, and epidural ropivacaine concentration $(p=0.03)$ as independent prognostic factors and higher dose of intraoperative epidural ropivacaine infusion remained as a significant protective factor in the model $(\mathrm{HR}=0.65,95 \% \mathrm{CI}, 0.44-0.94 ; \mathrm{p}=0.03)$.

Table 1. Characteristic of pancreatic cancer patients at baseline

\begin{tabular}{|c|c|c|c|c|}
\hline & \multicolumn{4}{|c|}{ Epidural Ropivacaine Concentration } \\
\hline & Total $(n=215)$ & High $(n=119)$ & Low $(n=96)$ & p-value \\
\hline \multicolumn{5}{|l|}{ Gender } \\
\hline Male & $125(58.1 \%)$ & $76(63.9 \%)$ & $49(51.0 \%)$ & \multirow[t]{2}{*}{$0.071 \#$} \\
\hline Female & $90(41.9 \%)$ & $43(36.1 \%)$ & $47(49.0 \%)$ & \\
\hline \multicolumn{5}{|l|}{ Age } \\
\hline Mean (SD) & $63.3( \pm 8.6)$ & $62.7( \pm 8.5)$ & $64.0( \pm 8.9)$ & $0.21^{*}$ \\
\hline \multicolumn{5}{|c|}{ Primary tumor location on pancreas } \\
\hline Head & $117(54.4 \%)$ & $71(59.7 \%)$ & $46(47.9 \%)$ & \multirow[t]{3}{*}{$0.083 \#$} \\
\hline Body \& Tail & $93(43.3 \%)$ & $44(37.0 \%)$ & $49(51.0 \%)$ & \\
\hline Total Pancreas & $5(2.3 \%)$ & $4(3.4 \%)$ & $1(1.0 \%)$ & \\
\hline \multicolumn{5}{|l|}{ AJCC 8th stage } \\
\hline Carcinoma in situ & $19(8.8 \%)$ & $11(9.2 \%)$ & $8(8.3 \%)$ & \multirow[t]{7}{*}{$0.4 \#$} \\
\hline IA & $32(14.9 \%)$ & $23(19.3 \%)$ & $9(9.4 \%)$ & \\
\hline IB & $60(27.9 \%)$ & $34(28.6 \%)$ & $26(27.1 \%)$ & \\
\hline IIA & $16(7.4 \%)$ & $9(7.6 \%)$ & $7(7.3 \%)$ & \\
\hline IIB & $70(32.6 \%)$ & $34(28.5 \%)$ & $36(37.5 \%)$ & \\
\hline III & $15(7.0 \%)$ & $7(5.9 \%)$ & $8(8.3 \%)$ & \\
\hline IV & $3(1.4 \%)$ & $1(0.8 \%)$ & $2(2.1 \%)$ & \\
\hline \multicolumn{5}{|l|}{ Primary tumor } \\
\hline Tis & $19(8.8 \%)$ & $11(9.2 \%)$ & $8(8.3 \%)$ & \multirow[t]{5}{*}{$0.29 \#$} \\
\hline $\mathrm{T} 1$ & $46(21.4 \%)$ & $31(26.1 \%)$ & $15(15.6 \%)$ & \\
\hline $\mathrm{T} 2$ & $117(54.4 \%)$ & $60(50.4 \%)$ & $57(59.4 \%)$ & \\
\hline T3 & $32(14.9 \%)$ & $16(13.4 \%)$ & $16(16.7 \%)$ & \\
\hline $\mathrm{T} 4$ & $1(0.5 \%)$ & $1(0.8 \%)$ & $0(0.0 \%)$ & \\
\hline \multicolumn{5}{|c|}{ Regional Lymph Nodes } \\
\hline N0 & $126(59.2 \%)$ & $77(65.3 \%)$ & $49(51.6 \%)$ & \multirow[t]{3}{*}{$0.13 \#$} \\
\hline N1 & $72(33.8 \%)$ & $34(28.8 \%)$ & $38(40.0 \%)$ & \\
\hline $\mathrm{N} 2$ & $15(7.0 \%)$ & $7(5.9 \%)$ & $8(8.4 \%)$ & \\
\hline \multicolumn{5}{|l|}{ Distant Metastasis } \\
\hline M0 & $212(98.6 \%)$ & $118(99.2 \%)$ & $94(97.9 \%)$ & \multirow[t]{2}{*}{$0.59 \#$} \\
\hline M1 & $3(1.4 \%)$ & $1(0.8 \%)$ & $2(2.1 \%)$ & \\
\hline \multicolumn{5}{|l|}{ Chemotherapy } \\
\hline No & $39(18.1 \%)$ & $20(16.8 \%)$ & $19(19.8 \%)$ & \multirow[t]{3}{*}{$0.77 \#$} \\
\hline Yes & $166(77.2 \%)$ & $94(79.0 \%)$ & $72(75.0 \%)$ & \\
\hline Unknown & $10(4.7 \%)$ & $5(4.2 \%)$ & $5(5.2 \%)$ & \\
\hline \multicolumn{5}{|l|}{ Radiotherapy } \\
\hline No & $174(80.9 \%)$ & $94(79.0 \%)$ & $80(83.3 \%)$ & $0.29 \#$ \\
\hline
\end{tabular}

\begin{tabular}{|c|c|c|c|c|}
\hline & \multicolumn{4}{|c|}{ Epidural Ropivacaine Concentration } \\
\hline & Total $(\mathrm{n}=215)$ & High $(\mathrm{n}=119)$ & Low $(n=96)$ & $\mathrm{p}$-value \\
\hline Yes & $40(18.6 \%)$ & $25(21.0 \%)$ & $15(15.6 \%)$ & \\
\hline Unknown & $1(0.5 \%)$ & $0(0.0 \%)$ & $1(1.0 \%)$ & \\
\hline \multicolumn{5}{|l|}{ CA 19-9 (U/ml) } \\
\hline$<35$ & $59(27.4 \%)$ & $32(26.9 \%)$ & $27(28.1 \%)$ & $0.64 \#$ \\
\hline $35-200$ & $72(33.5 \%)$ & $43(36.1 \%)$ & $29(30.2 \%)$ & \\
\hline$>200$ & $84(39.1 \%)$ & $44(37.0 \%)$ & $40(41.7 \%)$ & \\
\hline \multicolumn{5}{|l|}{ Microvascular Invasion } \\
\hline No & $189(87.9 \%)$ & $105(88.2 \%)$ & $84(87.5 \%)$ & $1 \#$ \\
\hline Yes & $26(12.1 \%)$ & $14(11.8 \%)$ & $12(12.5 \%)$ & \\
\hline \multicolumn{5}{|l|}{ Nerve Invasion } \\
\hline No & $63(29.3 \%)$ & $36(30.3 \%)$ & $27(28.1 \%)$ & $0.76 \#$ \\
\hline Yes & $152(70.7 \%)$ & $83(69.7 \%)$ & $69(71.9 \%)$ & \\
\hline \multicolumn{5}{|l|}{ Fat Invasion } \\
\hline No & $54(25.1 \%)$ & $31(26.1 \%)$ & $23(24.0 \%)$ & $0.75 \#$ \\
\hline Yes & $161(74.9 \%)$ & $88(73.9 \%)$ & $73(76.0 \%)$ & \\
\hline \multicolumn{5}{|l|}{ Resection Margin } \\
\hline Negative & $209(97.2 \%)$ & $115(96.6 \%)$ & $94(97.9 \%)$ & $0.69 \#$ \\
\hline Positive & $6(2.8 \%)$ & $4(3.4 \%)$ & $2(2.1 \%)$ & \\
\hline \multicolumn{5}{|l|}{ Tumor Deposits } \\
\hline No & $199(92.6 \%)$ & $107(89.9 \%)$ & $92(95.8 \%)$ & $0.12 \#$ \\
\hline Yes & $16(7.4 \%)$ & $12(10.1 \%)$ & $4(4.2 \%)$ & \\
\hline \multicolumn{5}{|l|}{ Tumor Differentiation } \\
\hline Well differentiated & $19(8.8 \%)$ & $11(9.2 \%)$ & $8(8.3 \%)$ & $0.37 \#$ \\
\hline Moderately differentiated & $78(35.9 \%)$ & $39(32.8 \%)$ & $39(40.6 \%)$ & \\
\hline Poorly differentiated & $118(54.9 \%)$ & $69(58.0 \%)$ & $49(51.0 \%)$ & \\
\hline \multicolumn{5}{|c|}{ Total Opioid Dose (Fentanyl, $\mu \mathrm{g})$} \\
\hline Mean (SD) & $190.5( \pm 71.1)$ & $180.0( \pm 67.3)$ & $198.9( \pm 73.3)$ & $0.049^{*}$ \\
\hline \multicolumn{5}{|l|}{ ASA score } \\
\hline 1 & $31(14.4 \%)$ & $15(12.6 \%)$ & $16(16.7 \%)$ & $0.73 \#$ \\
\hline 2 & $180(83.7 \%)$ & $101(84.9 \%)$ & $79(82.2 \%)$ & \\
\hline 3 & $4(1.9 \%)$ & $3(2.5 \%)$ & $1(1.0 \%)$ & \\
\hline \multicolumn{5}{|l|}{ Surgical Procedure } \\
\hline Open surgery & $215(100.0 \%)$ & $96(100.0 \%)$ & $119(100.0 \%)$ & 1 \\
\hline \multicolumn{5}{|c|}{ Clavien-Dindo Classification } \\
\hline$<3$ & $211(98.1 \%)$ & $95(99.0 \%)$ & $116(97.5 \%)$ & $0.63 \#$ \\
\hline$\geq 3$ & $4(1.9 \%)$ & $1(1.0 \%)$ & $3(2.5 \%)$ & \\
\hline \multicolumn{5}{|c|}{ Operation Duration (min) } \\
\hline Mean (SD) & $216.0( \pm 107.6)$ & $187.1( \pm 88.3)$ & $239.3( \pm 116.2)$ & $<0.001^{*}$ \\
\hline
\end{tabular}

\section{Exploratory subgroup analyses}

To further investigate whether epidural ropivacaine concentration was a prognostic factor in certain patient subgroups, we performed exploratory subgroup analyses. As shown in Figure 3, the forest plot demonstrated that high ropivacaine concentration had better prognostic potential in male (HR=0.71, 95\%CI: 0.51-0.99, $\mathrm{p}=0.042$ ) patients with ASA II $(\mathrm{HR}=0.74,95 \% \mathrm{CI}: 0.56-0.98, \mathrm{p}=0.038)$. PDAC patients with $\mathrm{T} 3$ stage disease $(\mathrm{HR}=0.3,95 \% \mathrm{CI}$ : $0.15-0.6, \mathrm{p}<0.001)$ and preoperative CA 19-9 higher than $200 \mathrm{U} / \mathrm{ml}(\mathrm{HR}=0.57,95 \% \mathrm{CI}: 0.38-0.84, \mathrm{p}=0.005)$ were more likely to benefit from higher dose ropivacaine. Meanwhile, high ropivacaine concentration remained as a positive prognostic factor in patients with negative resection margin $(\mathrm{HR}=0.76$, 95\%CI: 0.58-0.98, $\mathrm{p}=0.035)$, and without distant metastasis (HR=0.76, 95\%CI: 0.58-0.98, $\mathrm{p}=0.036)$, FI (HR=0.45, 95\%CI: 0.22-0.91, $\mathrm{p}=0.028$ ), tumor deposit ( $\mathrm{HR}=0.72,95 \% \mathrm{CI}: 0.55-0.95, \mathrm{p}=0.02)$, and adjuvant radiotherapy $(\mathrm{HR}=0.67,95 \% \mathrm{CI}: 0.5-0.9, \mathrm{p}=0.007)$. 

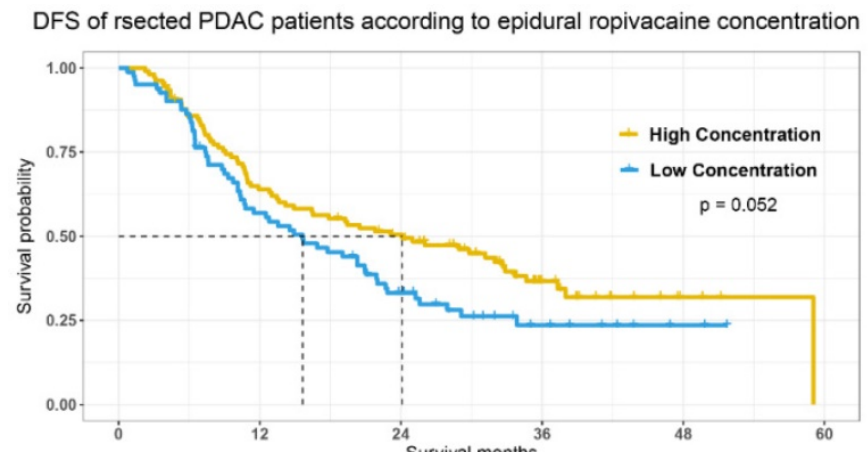

Number at risk: $\mathbf{n}(\%)$

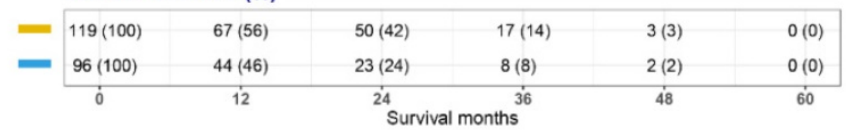

OS of rsected PDAC patients according to epidural ropivacaine concentration

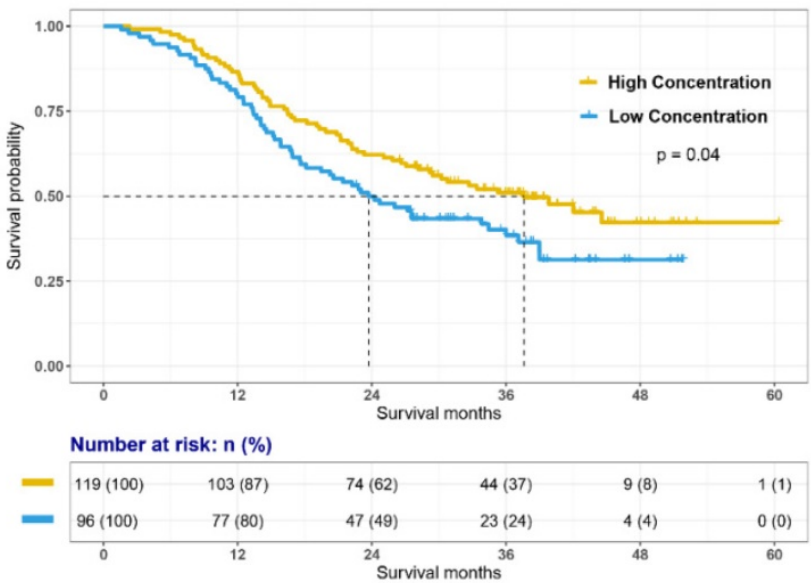

Figure 2. Disease free survival (DFS) and overall survival (OS) of pancreatic ductal adenocarcinoma (PDAC) patients undergoing pancreatectomy according to the epidural ropivacaine concentration they received.

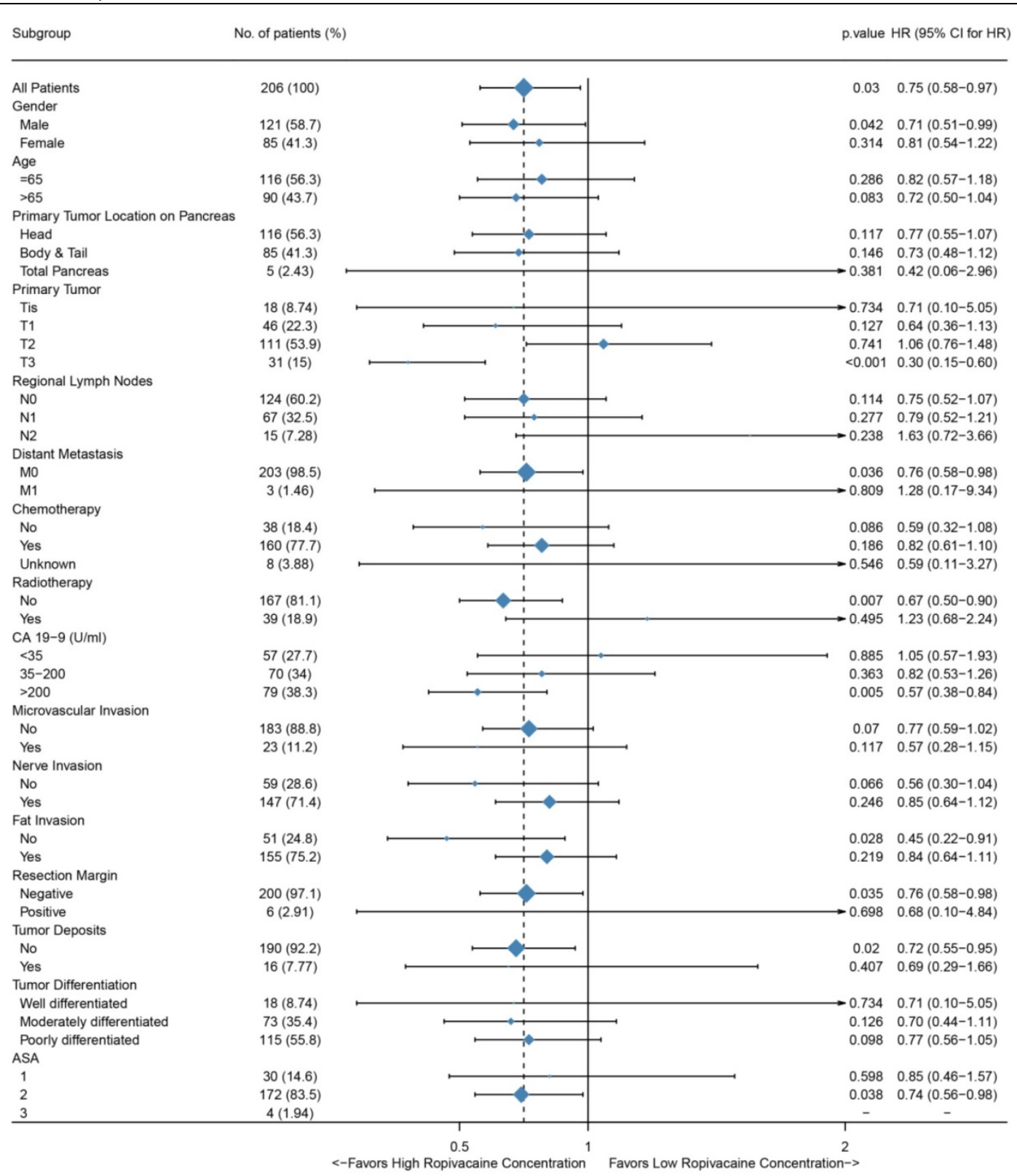

Figure 3. Forest plots of hazard ratios (HR) according to subgroup analysis for OS. The $\mathrm{x}$-axis shows the $\mathrm{HR}$ and $95 \%$ confidence interval (Cl) of each subgroup. The size of the boxes represents the relative number of patients in each subgroup. 
Table 2. Cox regression analysis for overall survival

\begin{tabular}{|c|c|c|c|c|}
\hline \multirow[t]{2}{*}{ Variables } & \multicolumn{2}{|l|}{ Univariate } & \multicolumn{2}{|l|}{ Multivariate } \\
\hline & HR & p-value & HR & p-value \\
\hline \multicolumn{5}{|l|}{ Gender } \\
\hline Male & 1 (as reference) & 0.803 & & \\
\hline Female & $0.955(0.664-1.37)$ & & & \\
\hline \multicolumn{5}{|l|}{ Age } \\
\hline Mean (SD) & $1.02(0.9982-1.042)$ & 0.073 & & \\
\hline \multicolumn{5}{|c|}{ Primary tumor location on pancreas } \\
\hline Head & 1 (as reference) & 0.58 & & \\
\hline Body \& Tail & $0.884(0.613-1.27)$ & 0.509 & & \\
\hline Others & $0.561(0.138-2.29)$ & 0.421 & & \\
\hline \multicolumn{5}{|l|}{ Primary Tumor } \\
\hline Tis & 1 (as reference) & $<0.01$ & 1 (as reference) & $<0.01$ \\
\hline $\mathrm{T} 1$ & $6.75(1.6-28.5)$ & $<0.01$ & $1.05(1.02-1.96)$ & $<0.01$ \\
\hline $\mathrm{T} 2$ & $9.02(2.21-36.8)$ & $<0.01$ & $1.36(1.21-1.52)$ & $<0.01$ \\
\hline $\mathrm{T} 3$ & $13(3.06-55.5)$ & $<0.01$ & $1.62(1.44-1.81)$ & $<0.01$ \\
\hline \multicolumn{5}{|c|}{ Regional Lymph Nodes } \\
\hline No & 1 (as reference) & $<0.01$ & 1 (as reference) & $<0.01$ \\
\hline N1 & $1.63(1.11-2.38)$ & 0.012 & $1.48(1.24-1.69)$ & $<0.01$ \\
\hline N2 & $2.92(1.56-5.45)$ & $<0.01$ & $1.72(1.41-1.99)$ & $<0.01$ \\
\hline \multicolumn{5}{|l|}{ Distant Metastasis } \\
\hline M0 & 1 (as reference) & 0.019 & 1 (as reference) & $<0.01$ \\
\hline M1 & $5.79(1.82-18.4)$ & & $3.72(2.71-5.28)$ & \\
\hline \multicolumn{5}{|l|}{ Chemotherapy } \\
\hline No & 1 (as reference) & 0.04 & 1 (as reference) & $<0.01$ \\
\hline Yes & $0.672(0.429-0.924)$ & & $0.35(0.21-0.59)$ & \\
\hline \multicolumn{5}{|l|}{ Radiotherapy } \\
\hline No & 1 (as reference) & 0.436 & & \\
\hline Yes & $1.19(0.77-1.84)$ & & & \\
\hline \multicolumn{5}{|l|}{ CA $19-9(\mathrm{U} / \mathrm{ml})$} \\
\hline$<35$ & 1 (as reference) & $<0.01$ & 1 (as reference) & $<0.01$ \\
\hline $35-200$ & $1.74(1.04-2.91)$ & 0.036 & $1.27(0.74-2.28)$ & 0.322 \\
\hline$>200$ & $2.42(1.48-3.95)$ & $<0.01$ & $1.76(1.04-2.99)$ & 0.031 \\
\hline \multicolumn{5}{|c|}{ Microvascular Invasion } \\
\hline No & 1 (as reference) & 0.313 & & \\
\hline Yes & $1.3(0.79-2.15)$ & & & \\
\hline \multicolumn{5}{|l|}{ Nerve Invasion } \\
\hline No & 1 (as reference) & $<0.01$ & 1 (as reference) & 0.28 \\
\hline Yes & $2.47(1.54-3.96)$ & & $1.33(0.78-2.28)$ & \\
\hline \multicolumn{5}{|l|}{ Fat Invasion } \\
\hline No & 1 (as reference) & $<0.01$ & 1 (as reference) & 0.08 \\
\hline Yes & $2.78(1.66-4.64)$ & & $1.61(0.941-2.98)$ & \\
\hline Resection Margin & & & & \\
\hline Negative & 1 (as reference) & 0.252 & & \\
\hline Positive & $0.485(0.12-1.96)$ & & & \\
\hline Tumor Deposits & & & & \\
\hline No & 1 (as reference) & $<0.01$ & 1 (as reference) & 0.289 \\
\hline Yes & $2.37(1.32-4.23)$ & & $1.44(0.73-2.92)$ & \\
\hline Tumor Differentiatio & & & & \\
\hline Well differentiated & 1 (as reference) & $<0.01$ & 1 (as reference) & 0.042 \\
\hline $\begin{array}{l}\text { Moderately } \\
\text { differentiated }\end{array}$ & $6.52(1.57-27.1)$ & $<0.01$ & $2(0.12-34.1)$ & 0.616 \\
\hline $\begin{array}{l}\text { Poorly differentiated } \\
\text { ASA }\end{array}$ & $10.8(2.65-44)$ & $<0.01$ & $2.55(0.122-46.9)$ & 0.45 \\
\hline 1 & 1 (as reference) & 0.078 & & \\
\hline 2 & $0.748(0.466-1.2)$ & 0.229 & & \\
\hline 3 & $0.275(0.037-2.05)$ & 0.208 & & \\
\hline Total Opioid Dose & entanyl, $\mu g)$ & & & \\
\hline Mean (SD) & $0.998(0.994-1.004)$ & 0.138 & & \\
\hline Clavien-Dindo Clas & fication & & & \\
\hline$<3$ & 1 (as reference) & 0.836 & & \\
\hline$\geq 3$ & $0.863(0.213-3.49)$ & & & \\
\hline Epidural Ropivacai & Concentration & & & \\
\hline Low & 1 (as reference) & 0.03 & 1 (as reference) & 0.03 \\
\hline High & $0.75(0.58-0.97)$ & & $0.65(0.44-0.94)$ & \\
\hline
\end{tabular}

The prognostic value of epidural ropivacaine concentration in these subgroups was further confirmed by the survival analyses showed in Figure 4 and supplemental Figure 1. The results indicated the possible prognostic effect of epidural ropivacaine concentration in PDAC patients with T3M0 disease, preoperative CA19-9 higher than $200 \mathrm{U} / \mathrm{ml}$, negative resection margin, and those without tumor deposit and adjuvant radiotherapy.

\section{Discussion}

In the present study, we investigated the association of intraoperative epidural administration of ropivacaine and survival outcome of PDAC patients who received curative surgery. Survival analyses showed that patients who received $0.375 \%-0.5 \%$ ropivacaine through the epidural catheter intraoperatively had better OS. Meanwhile, high epidural ropivacaine concentration was an independent protective factor in Cox proportional hazards model. Further subgroup analyses demonstrated the possible prognostic effect of epidural ropivacaine concentration in T3M0 PDAC patients with preoperative CA 19-9 higher than 200 $\mathrm{U} / \mathrm{ml}$, negative resection margin, and those without tumor deposit and adjuvant radiotherapy.

In recent years, the effect of intraoperative anesthetic management on long-term clinical outcome of cancer patients has become an interesting research topic. Several retrospective studies reported that local anesthesia, especially perioperative epidural analgesia, was related to better survival and a lower incidence of local recurrence or distant metastasis in cancer patients [5-7,18-21]. Tyler et al. observed the association between use of epidural anesthesia during primary pancreatic cancer resection and improved survival among PDAC patients [9]. This was consistent with our present study which demonstrated that PDAC patients who intraoperatively received $0.375 \%-0.5 \%$ ropivacaine through epidural catheter had better OS.

There is not standard institutional protocol for ropivacaine dose selection in our medical center. However, as mentioned above, we prefer to deliver higher dose of ropivacaine for younger patients receiving radical and time-consuming surgeries to reduce potential perioperative complications and to provide better abdominal muscles relaxation. These were consistent with our analyses in Table 1. Patients in high ropivacaine concentration group had longer operation duration. Also, patients receiving higher dose of ropivacaine seemed to be younger and had higher proportion of pancreatic head tumor, and thus had more pancreaticoduodenectomy procedures. Again, more complicated surgical procedures and longer operation time could also explain increased dosage of opioid in high ropivacaine concentration 
group.

Interestingly, we found that PDAC patients with T3M0 disease and preoperative CA 19-9 level higher than $200 \mathrm{U} / \mathrm{ml}$, negative resection margin, and those without tumor deposit and adjuvant radiotherapy were more likely to benefit from high concentration of ropivacaine. As shown in Table 2, both T3 and preoperative CA 19-9 higher than $200 \mathrm{U} / \mathrm{ml}$ were
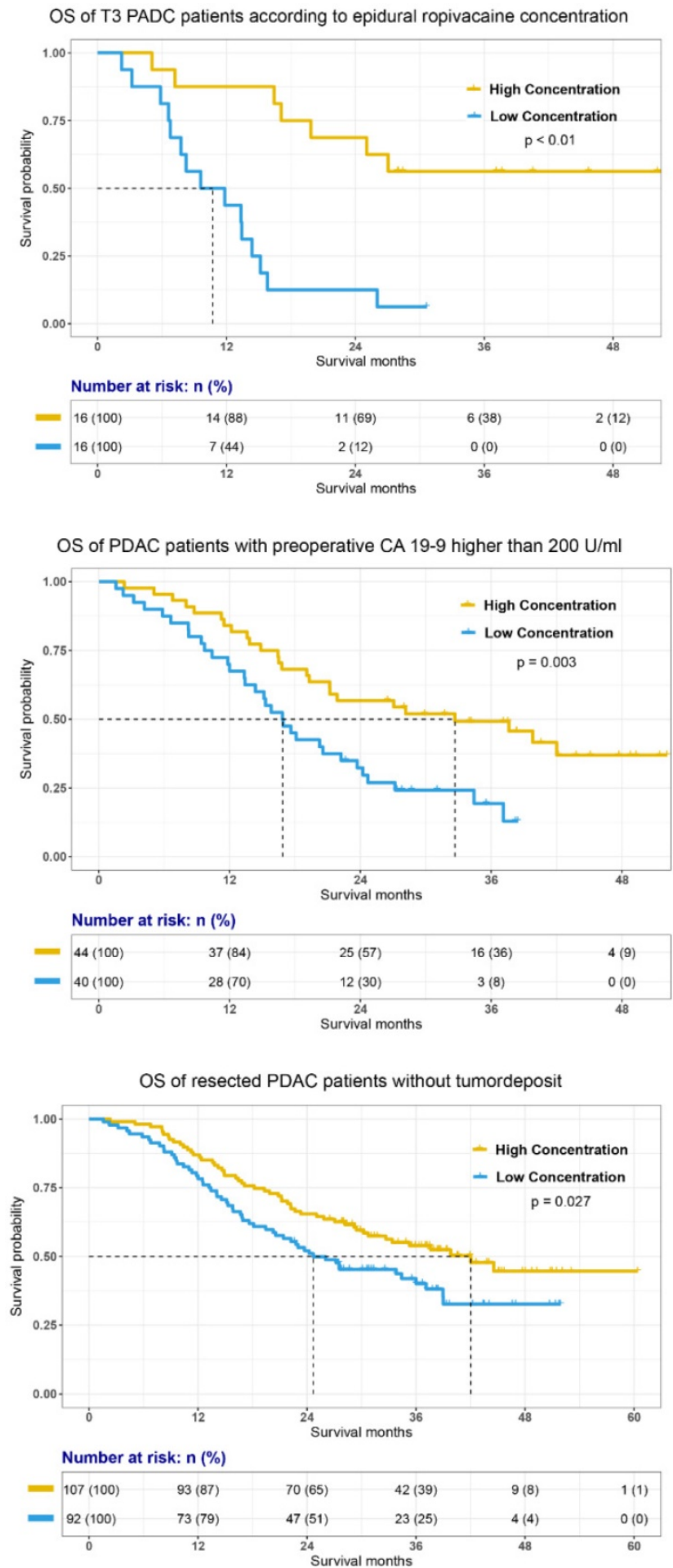

negative prognostic factors for PDAC patients (T3, $\mathrm{HR}=1.57$, 95\%CI: 1.44-1.72, p<0.01; CA 19-9 > 200 $\mathrm{U} / \mathrm{ml}, \mathrm{HR}=1.76,95 \% \mathrm{CI}$ : 1.04-2.99, $\mathrm{p}=0.035)$. With manageable side effects of analgesics, further clinical research was needed to investigate whether higher epidural ropivacaine concentration could provide survival benefits for PDAC patients, especially for those with preoperative CA 19-9 higher than 200
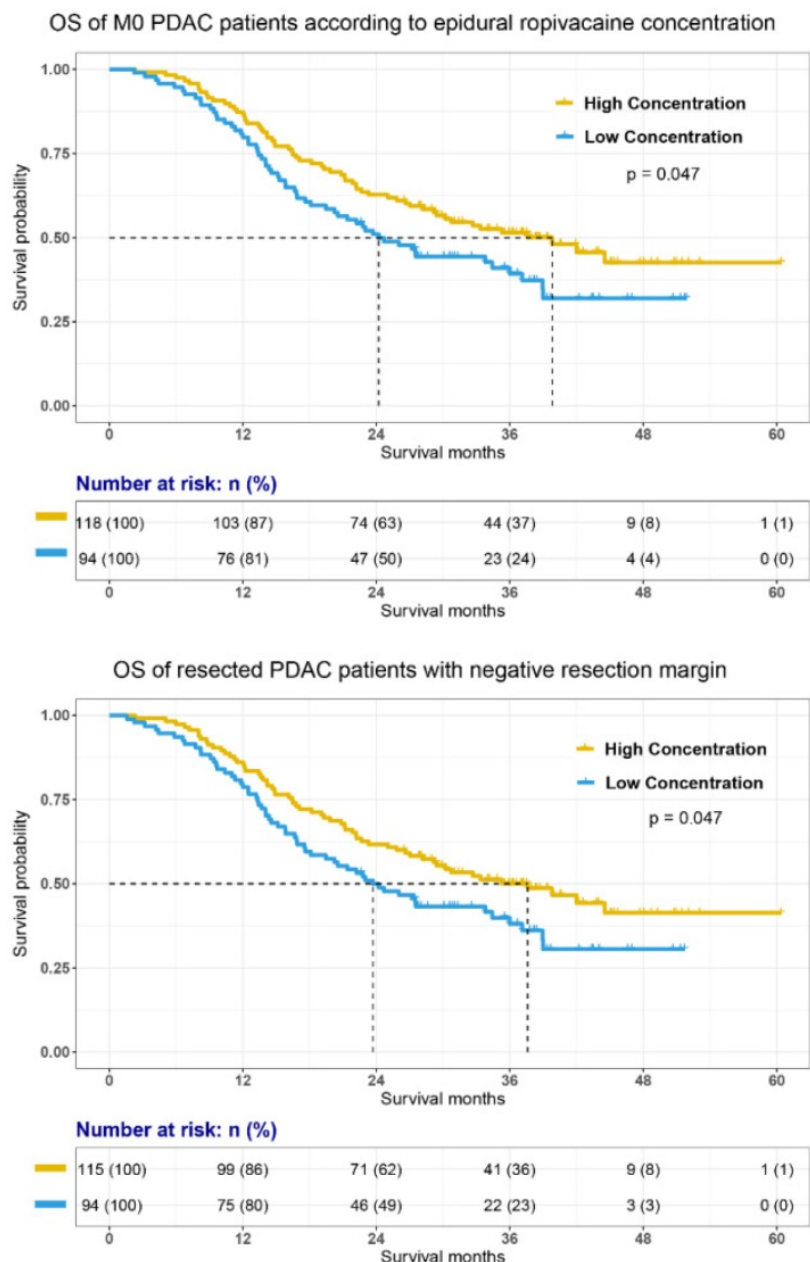

OS of resected PDAC patients without adjuvant radiotherapy

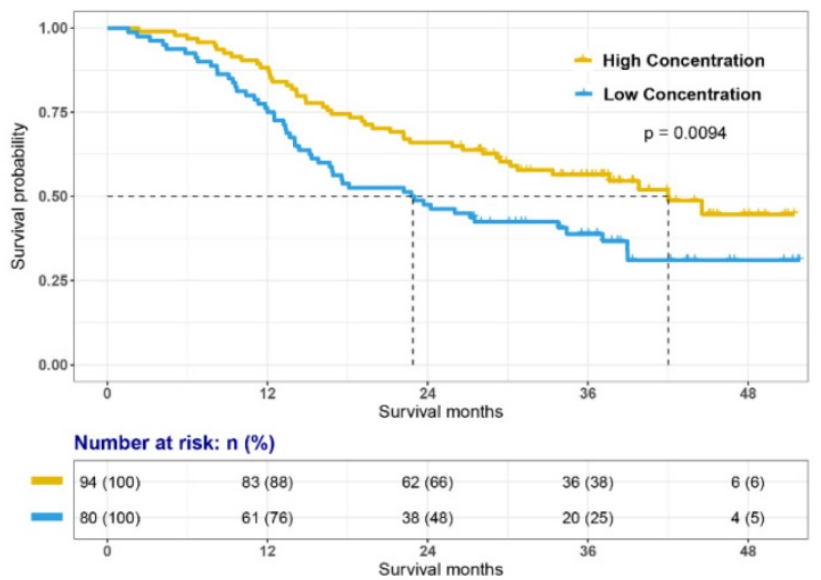

Figure 4. Survival analyses of specific subgroups according to the epidural ropivacaine concentration. 
$\mathrm{U} / \mathrm{ml}$, and those whose preoperative imaging assessment showed that primary pancreatic tumor was $>4 \mathrm{~cm}$ in greatest dimension without distant metastasis.

In Table 1, we found that PDAC patients in $0.375 \%-0.5 \%$ ropivacaine group received increased opioid dosing and Cox proportional model demonstrated that total opioid dose was not a prognostic factor. However, previous literatures showed that reduced opioid dosing would potentially improve perioperative immune function and postoperative long-term survival [22,23]. Thus, difference in opioid dose may not account for survival benefit of high dose ropivacaine.

Previous literatures have demonstrated that local anesthetics exerted cytotoxic effect on neural cells in a concentration-dependent fashion [24,25]. Meanwhile, ropivacaine was proven to inhibit lung and breast cancer cell proliferation by inducing cell cycle arrest in vitro [6,7]. In addition, local-anesthetic-sensitive voltage-gated ion channels functionally expressed in a variety of carcinomas and also lymphocytes, and have shown to enhance metastatic cell behaviors [26-30]. Accumulating evidences have demonstrated that ropivacaine could block voltage-gated ion channels activity, and thus suppress invasiveness of cancer cells and directly affect the local immune response to surgery $[10,26,31]$. Meanwhile, we found the trend that patients in high concentration group seemed to achieve better DFS in the present study. Thus, the unexpected survival benefit of high concentration ropivacaine may be due to its inhibitory effect on cancer cell proliferation and migration. However, these effects of ropivacaine were shown only in vitro. The translation of these effects into clinical outcomes remained unclear.

Several important limitations are inherent in this study. First, this was a retrospective study associating with bias and confounding owing to unmeasured variables that might influence the survival outcome. Second, we did not include preoperative nutritional status and details of adjuvant therapy (e.g. chemotherapy regimen and duration of chemotherapy) in the study. However, these variables might have a significant impact on primary endpoint. Third, our results relate to only ropivacaine epidural anesthesia and do not translate to other neuraxial anesthetic techniques. The association between anesthetic techniques and oncologic outcomes among patients with pancreatic cancer need to be better characterized from a pathophysiological standpoint.

In conclusion, resected PDAC patients who received high concentration of ropivacaine through the epidural catheter intraoperatively had improved OS. Further prospective study is needed to investigate the impact of local anesthesia on oncology outcome of PDAC patients who received primary tumor resection.

\section{Supplementary Material}

Supplementary figure.

http://www.jcancer.org/v12p4513s1.pdf

\section{Acknowledgements}

This work was supported by Science and Technology Commission of Shanghai Municipality [grant number 17XD1401200] and the Open Fund of Key Laboratory of Hepatosplenic Surgery, Ministry of Education, Harbin, China [grant number GPKF202009].

\section{Competing Interests}

The authors have declared that no competing interest exists.

\section{References}

1. Siegel RL, Miller KD, Jemal A. Cancer statistics, 2020. CA Cancer J Clin. 2020; 70: 7-30.

2. Wu W, Jin G, Wang C, et al. The current surgical treatment of pancreatic cancer in China: a national wide cross-sectional study. Journal of Pancreatology. 2019; 2.

3. Wigmore TJ, Mohammed K, Jhanji S. Long-term Survival for Patients Undergoing Volatile versus IV Anesthesia for Cancer Surgery: A Retrospective Analysis. ANESTHESIOLOGY. 2016; 124: 69-79.

4. Lai HC, Lee MS, Lin KT, et al. Propofol-based total intravenous anesthesia is associated with better survival than desflurane anesthesia in robot-assisted radical prostatectomy. PLOS ONE. 2020; 15: e230290.

5. Chipollini J, Alford B, Boulware DC, et al. Epidural anesthesia and cancer outcomes in bladder cancer patients: is it the technique or the medication? A matched-cohort analysis from a tertiary referral center. BMC ANESTHESIOL. 2018; 18: 157.

6. Li R, Xiao C, Liu H, et al. Effects of local anesthetics on breast cancer cell viability and migration. BMC CANCER. 2018; 18: 666.

7. Wang HW, Wang LY, Jiang L, et al. Amide-linked local anesthetics induce apoptosis in human non-small cell lung cancer. J THORAC DIS. 2016; 8: 2748-2757

8. Tedore T. Regional anaesthesia and analgesia: relationship to cancer recurrence and survival. Br J Anaesth. 2015; 115 Suppl 2: i34-i45.

9. Call TR, Pace NL, Thorup DB, et al. Factors associated with improved survival after resection of pancreatic adenocarcinoma: a multivariable model. ANESTHESIOLOGY. 2015; 122: 317-324.

10. Baptista-Hon DT, Robertson FM, Robertson GB, et al. Potent inhibition by ropivacaine of metastatic colon cancer SW620 cell invasion and NaV1.5 channel function. Br J Anaesth. 2014; 113 Suppl 1: i39-i48.

11. Aldakkak M, Christians KK, Krepline AN, et al. Pre-treatment carbohydrate antigen 19-9 does not predict the response to neoadjuvant therapy in patients with localized pancreatic cancer. HPB (Oxford). 2015; 17: 942-952.

12. Hong JM, Lee $\mathrm{HJ}$, Oh $\mathrm{YJ}$, et al. Observations on significant hemodynamic changes caused by a high concentration of epidurally administered ropivacaine: correlation and prediction study of stroke volume variation and central venous pressure in thoracic epidural anesthesia. BMC ANESTHESIOL. 2017; 17: 153.

13. Zink W, Graf BM. The toxicity of local anesthetics: the place of ropivacaine and levobupivacaine. Curr Opin Anaesthesiol. 2008; 21: 645-650.

14. Rioja E, Rubio-Martinez LM, Monteith G, et al. Analgesic and motor effects of a high-volume intercoccygeal epidural injection of $0.125 \%$ or $0.0625 \%$ bupivacaine in adult cows. CAN J VET RES. 2013; 77: 273-280.

15. Pathak A, Yadav N, Mohanty SN, et al. Comparison of Three Different Concentrations $0.2 \%, 0.5 \%$, and $0.75 \%$ Epidural Ropivacaine for Postoperative Analgesia in Lower Limb Orthopedic Surgery. Anesth Essays Res. 2017; 11: 1022-1025.

16. I McConachie, J McGeachie, J Barrie. (2003). Wylie and Churchill Davidson's A Practice of Anesthesia. (Vol. 37). London: Hodder Education Publishers.

17. Capogna G, Camorcia M, Columb MO. Minimum analgesic doses of fentanyl and sufentanil for epidural analgesia in the first stage of labor. ANESTH ANALG. 2003; 96: 1178-1182. 
18. Biki B, Mascha E, Moriarty DC, et al. Anesthetic technique for radical prostatectomy surgery affects cancer recurrence: a retrospective analysis. ANESTHESIOLOGY. 2008; 109: 180-187.

19. Exadaktylos AK, Buggy DJ, Moriarty DC, et al. Can anesthetic technique for primary breast cancer surgery affect recurrence or metastasis? ANESTHESIOLOGY. 2006; 105: 660-664.

20. Snyder GL, Greenberg S. Effect of anaesthetic technique and other perioperative factors on cancer recurrence. Br J Anaesth. 2010; 105: 106-115.

21. Sessler DI, Ben-Eliyahu S, Mascha EJ, et al. Can regional analgesia reduce the risk of recurrence after breast cancer? Methodology of a multicenter randomized trial. CONTEMP CLIN TRIALS. 2008; 29: 517-526.

22. Oh TK, Do SH, Yoon YS, et al. Association between Opioid use and Survival time in patients with Unresectable Pancreatic Cancer: 10 Years of Clinical Experience. PANCREAS. 2018; 47: 837-842.

23. Novy DM, Nelson DV, Koyyalagunta D, et al. Pain, opioid therapy, and survival: a needed discussion. PAIN. 2020; 161: 496-501.

24. Werdehausen R, Fazeli S, Braun S, et al. Apoptosis induction by different local anaesthetics in a neuroblastoma cell line. Br J Anaesth. 2009; 103: 711-718.

25. Perez-Castro R, Patel S, Garavito-Aguilar ZV, et al. Cytotoxicity of local anesthetics in human neuronal cells. ANESTH ANALG. 2009; 108: 997-1007.

26. Lo WL, Donermeyer DL, Allen PM. A voltage-gated sodium channel is essential for the positive selection of CD4(+) T cells. NAT IMMUNOL. 2012; 13: 880-887.

27. Laniado ME, Lalani EN, Fraser SP, et al. Expression and functional analysis of voltage-activated $\mathrm{Na}+$ channels in human prostate cancer cell lines and their contribution to invasion in vitro. AM J PATHOL. 1997; 150: 1213-1221.

28. Brisson L, Gillet L, Calaghan $\mathrm{S}$, et al. $\mathrm{Na}(\mathrm{V}) 1.5$ enhances breast cancer cell invasiveness by increasing NHE1-dependent $\mathrm{H}(+)$ efflux in caveolae. ONCOGENE. 2011; 30: 2070-2076.

29. House CD, Vaske CJ, Schwartz AM, et al. Voltage-gated Na+ channel SCN5A is a key regulator of a gene transcriptional network that controls colon cancer invasion. CANCER RES. 2010; 70: 6957-6967.

30. Fraser SP, Diss JK, Chioni AM, et al. Voltage-gated sodium channel expression and potentiation of human breast cancer metastasis. CLIN CANCER RES. 2005; 11: 5381-5389.

31. Fraser SP, Foo I, Djamgoz MB. Local anaesthetic use in cancer surgery and disease recurrence: role of voltage-gated sodium channels? Br J Anaesth. 2014; 113: 899-902. 